\title{
Combination of serum TIMP-3, CA125, and NT-proBNP in predicting ventricular remodeling in patients with heart failure following acute myocardial infarction
}

\author{
Yue-Tao Xie ${ }^{1}$, Yi Dang ${ }^{1}$, Fei-Fei Zhang ${ }^{1}$, Qian-Hui Zhang ${ }^{1}$, Hai-Bo Wu ${ }^{1}$, Guang Liu ${ }^{2}$ \\ ${ }^{1}$ Department of Cardiology, Hebei General Hospital, Shijiazhuang, China; ${ }^{2}$ Department of Cardiology, The Fourth Hospital of Hebei Medical \\ University, Shijiazhuang, China \\ Contributions: (I) Conception and design: YT Xie, HB Wu, G Liu; (II) Administrative support: HB Wu, Y Dang; (III) Provision of study materials \\ or patients: YT Xie, HB Wu, G Liu; (IV) Collection and assembly of data: FF Zhang, QH Zhang, YT Xie; (V) Data analysis and interpretation: FF \\ Zhang, QH Zhang, G Liu; (VI) Manuscript writing: All authors; (VII) Final approval of manuscript: All authors. \\ Correspondence to: Hai-Bo Wu, MD. Department of Cardiology, Hebei General Hospital, No. 348, Peace West Road, Shijiazhuang 050000, China. \\ Email: wuhb929@163.com; Guang Liu, MD. Department of Cardiology, The Fourth Affiliated Hospital of Hebei Medical University, No. 12, \\ Jiankang Road, Shijiazhuang 050000, China. Email: minewuhaibo@126.com.
}

Background: Left ventricular remodeling is the basic pathological mechanism of heart failure following acute
myocardial infarction (AMI). Determining sensitive indexes for the early prediction of ventricular remodeling
is important for the prevention of heart failure. This study aims to investigate the value of serum TIMP-3,
CA125, and NT-proBNP in predicting ventricular remodeling in patients with heart failure following AMI.
Methods: From May 2017 to May 2018, 93 patients with heart failure following AMI were enrolled in the
study. The participants were divided into two groups: the ventricular remodeling group (n=51) and the non-
ventricular remodeling group (n=42). In addition, 47 healthy subjects who underwent physical examinations
in the same period were enrolled as controls. Serum TIMP-3, CA125, and NT-proBNP were measured,
in addition to the left ventricular wall thickness (LVWT) and left ventricular mass index (LVMI). The
correlation of serum TIMP-3, CA125, and NT-proBNP with the LVWT and LVMI was analyzed, and its
value in predicting ventricular remodeling was evaluated. Results: Serum TIMP-3 level was lower $(\mathrm{P}<0.05)$ and CA125 and NT-proBNP levels were higher $(\mathrm{P}<0.05)$ in both the ventricular remodeling and non-ventricular remodeling groups compared with the control group. Furthermore, the serum TIMP-3 level was lower in the ventricular remodeling group compared with the non-ventricular remodeling group $(\mathrm{P}<0.05)$, while the levels of CA125 and NT-proBNP were higher in the ventricular remodeling group compared with the non-ventricular remodeling group $(\mathrm{P}<0.05)$. The serum TIMP-3 level was negatively correlated with the LVWT and LVMI, while serum CA125 and NT-proBNP levels were positively correlated with the LVWT and LVMI, respectively. The area under the receiver operating characteristic curve of the combination of serum TIMP-3, CA125, and NT-proBNP levels in predicting ventricular remodeling was 0.850 , and the prediction sensitivity and specificity were $74.51 \%$ and $87.71 \%$, respectively.

Conclusions: The combination of serum TIMP-3, CA125, and NT-proBNP can improve the sensitivity and specificity of predicting ventricular remodeling and can aid in the early prevention and treatment of heart failure.

Keywords: Acute myocardial infarction (AMI); heart failure; tissue inhibitor of matrix metalloproteinase 3; carbohydrate antigen CA125; N-terminal pro-brain natriuretic peptide (NT-proBNP)

Submitted Mar 30, 2020. Accepted for publication Jul 31, 2020.

doi: $10.21037 / \mathrm{cdt}-20-399$

View this article at: http://dx.doi.org/10.21037/cdt-20-399 


\section{Introduction}

Acute myocardial infarction (AMI) usually involves acute myocardial ischemic necrosis caused by secondary thrombosis as a result of coronary artery disease, which has a high mortality rate. The survival rate of AMI has significantly improved in recent years in cases of early treatment as a result of technological innovations and the development of medical technology. However, the incidence of combined heart failure is increasing. Combined heart failure develops rapidly and has a high mortality rate. Hence, it has become a serious condition that threatens the lives and health of patients (1).

Left ventricular remodeling is the basic pathological mechanism of heart failure following AMI and significantly increases the incidence of cardiac death. For this reason, determining sensitive indexes for the early prediction of ventricular remodeling and inhibiting ventricular remodeling are key bases for preventing and treating acute post-infarction heart failure (2). Tissue inhibitor of metalloproteinase-3 (TIMP-3) is a specific inhibitor of matrix metalloproteinase (MMP) activity and plays an important role in vascular remodeling and cardiovascular metabolism diseases (3). As biomarkers for heart failure, carbohydrate antigen CA125 and N-terminal pro-brain natriuretic peptide (NT-proBNP) have predictive value for the mortality risk of acute heart failure $(4,5)$. However, research exploring their value in predicting early ventricular remodeling remains scarce.

In the present study, serum TIMP-3, CA125, and NTproBNP levels in patients with AMI complicated with heart failure were measured, and the values of the single and combined uses of these indicators in predicting ventricular remodeling were analyzed to provide a reference for the early prevention and treatment of heart failure. We present the following article in accordance with the TRIPOD reporting checklist (available at http://dx.doi.org/10.21037/ cdt-20-399).

\section{Methods}

\section{General information}

The present research was a retrospective cohort study. A total of 93 AMI patients with heart failure who were treated in our hospital from May 2017 to May 2018 were enrolled in the study. Forty-seven healthy subjects who underwent physical examinations during the same period were enrolled as controls. The study was conducted in accordance with the Declaration of Helsinki (as was revised in 2013). The study was approved by institutional ethics board of Hebei General Hospital [2017 Scientific Research Proceedings No. (144)], and informed consent was provided by all patients.

The inclusion criteria for patients were as follows: (I) patients met the Guidelines for the Diagnosis and Treatment of Acute ST-segment Elevation Myocardial Infarction with chest pain for more than 30 minutes; abnormally elevated myocardial injury markers were present, such as creatine kinase and troponin; and there was electrocardiogram (ECG) ST segment elevation $>0.1 \mathrm{mv}$ in two or more adjacent leads (6). (II) Patients met the postinfarction heart failure diagnostic criteria. According to the ESC Guidelines for the Diagnosis and Treatment of Acute ST-segment Elevation Myocardial Infarction 2017, the patients were at Killip grades II, III, and IV (7). (III) Subject participation was approved by the Ethics Committees of our hospitals and informed consent was provided by the patients and their families. The exclusion criteria were as follows: (I) patients with a previous history of myocardial infarction, cardiac insufficiency, and heart related diseases; (II) patients with other with severe diabetes, malignant tumors, and infectious diseases; (III) patients with dysfunction of the liver, kidney, thyroid, or other organs; (IV) patients with connective tissue and/or autoimmune diseases.

\section{Detection of serum indices}

Twenty-four hours following admission, in a fasted state on the morning of the day following physical examination, $5 \mathrm{~mL}$ blood was drawn from the elbow vein of each AMI patient with heart failure, and $5 \mathrm{~mL}$ blood from the elbow vein was also drawn from all members of the control group. The serum was separated within two hours after blood sampling and was reserved by cryopreservation. Serum TIMP-3 levels were detected using an enzymelinked immunosorbent assay (ELISA) purchased from Yuanmush Biotech (Shanghai, China). Serum CA125 levels were detected by electrochemiluminescence. The CA125 quantitative test kit (chemiluminescence) was purchased from Relia Biotechnology (Jiangsu) Co., Ltd. Serum NTproBNP levels were detected by immunofluorescence. The VIDAS NT-proBNP kit was purchased from Relia Biotechnology (Jiangsu) Co., Ltd, and the automatic biochemical analyzer was obtained from Backman Coulter (China) Co., Ltd. 
Table 1 Comparison of general data among the three groups

\begin{tabular}{lcccccccc}
\hline \multirow{2}{*}{ Groups } & $\begin{array}{c}\text { Number } \\
\text { of cases }\end{array}$ & $\begin{array}{c}\text { Gender } \\
(\mathrm{M} / \mathrm{F})\end{array}$ & Age, years & $\begin{array}{c}\text { Hypertension, } \\
\mathrm{n}(\%)\end{array}$ & $\begin{array}{c}\text { Diabetes } \\
\text { mellitus, } \mathrm{n}(\%)\end{array}$ & $\begin{array}{c}\text { Smoking, } \\
\mathrm{n}(\%)\end{array}$ & LVWT (mm) & LVMI (g/m²) \\
\hline Control group & 47 & $28 / 19$ & $62.19 \pm 14.05$ & - & - & $17(36.17)$ & $8.76 \pm 1.98$ & $114.32 \pm 28.44$ \\
Non-remodeling group & 42 & $22 / 20$ & $60.97 \pm 12.54$ & $18(42.86)$ & $10(23.81)$ & $19(45.24)$ & $10.37 \pm 3.11^{*}$ & $127.73 \pm 30.25^{\star}$ \\
Remodeling group & 51 & $30 / 21$ & $61.48 \pm 13.26$ & $25(49.02)$ & $14(27.45)$ & $29(56.86)$ & $12.14 \pm 3.85^{\star \#}$ & $152.21 \pm 41.43^{* \#}$ \\
$\chi^{2} / \mathrm{F}$ & & 0.367 & 0.095 & 0.352 & 0.160 & 4.245 & 14.557 & 15.482 \\
P & & 0.832 & 0.910 & 0.553 & 0.690 & 0.120 & 0.000 & 0.000 \\
\hline
\end{tabular}

To compared with the control group, ${ }^{*} \mathrm{P}<0.05$; to compared with the non-remodeling group, ${ }^{\#} \mathrm{P}<0.05$. LVWT, left ventricular wall thickness; LVMI, left ventricular mass index.

\section{Ultrasound detection and grouping}

The left ventricular mass index (LVMI) was calculated. An increase of $\geq 20 \%$ of the left ventricular end-diastolic volume (LVEDV) at the sixth month after onset compared with the third day after onset was suggestive of left ventricular remodeling, while an increase of $<20 \%$ suggested non-left ventricular remodeling.

\section{Statistics analysis}

Statistical analysis was conducted using statistical software SPSS 22.0. Measurement data were expressed as mean \pm standard deviation (mean $\pm \mathrm{SD}$ ). Data were compared among the three groups using a one-way analysis of variance and between two groups using a $t$-test. Count data were expressed as a percentage and were compared among multiple groups and between two groups using an $\chi^{2}$ test. The predictive values of serum indices on ventricular remodeling were analyzed through the receiver operator characteristic (ROC) curve, and the ventricular remodeling risk factors were analyzed using logistic regression analysis. $\mathrm{P}<0.05$ was considered statistically significant.

\section{Results}

\section{Comparison of general information}

A total of 93 AMI patients with heart failure were enrolled in the study. Of these patients, 56 were male, and 37 were female. The age of the patients ranged from 42-78 years, with an average age of $61.42 \pm 13.36$ years. In terms of the Killip classification, 26 patients were at grade II, 31 patients were at grade III, and 36 patients were at grade IV. Fortyseven healthy subjects were enrolled as controls. Of these subjects, 28 were male, and 19 were female. The age of the subjects in the control group ranged from $40-80$ years, and the average age was $62.19 \pm 14.05$ years.

Patients with heart failure following AMI were examined by ultrasonography, and the LVEDV values were compared. Fifty-one patients exhibited left ventricular remodeling (including 8 patients at Killip II, 17 patients at Killip III, and 26 patients at Killip IV), while 42 patients exhibited no left ventricular remodeling. Differences in general information between the control group, non-ventricular remodeling group, and ventricular remodeling group, including gender, age, and smoking status, were not statistically significant $(\mathrm{P}>0.05)$. Furthermore, differences between the nonventricular remodeling group and ventricular remodeling group in underlying diseases, such as hypertension and diabetes mellitus, were not statistically significant $(\mathrm{P}>0.05)$. The left ventricular wall thickness (LVWT) and LVMI were significantly higher in the ventricular remodeling group than in the non-ventricular remodeling and control groups $(\mathrm{P}<0.05)$, and the LVWT and LVMI were significantly higher in the non-ventricular remodeling group than in the control group $(\mathrm{P}<0.05 ;$ Table 1$)$.

\section{Comparison of serum indexes}

Compared with the control group, serum TIMP-3 levels were lower in the ventricular remodeling and nonventricular remodeling groups, while the CA125 and NTproBNP levels were higher. Serum TIMP-3 levels were lower in the ventricular remodeling group than in the non-ventricular remodeling group, while CA125 and NTproBNP levels were higher in the ventricular remodeling group than in the non-ventricular remodeling group. The differences in serum TIMP-3, CA125, and NT-proBNP levels among the three groups were statistically significant $(\mathrm{P}<0.05$; Table 2). 
Table 2 Comparison of serum indices among the three groups

\begin{tabular}{lcccc}
\hline Groups & Number of cases & TIMP-3 (ng/L) & CA125 (IU/mL) & NT-proBNP (ng/L) \\
\hline Control group & 47 & $124.53 \pm 36.61$ & $22.86 \pm 6.43$ & $174.53 \pm 55.67$ \\
Non-remodeling group & 42 & $75.62 \pm 19.74^{\star}$ & $72.44 \pm 18.62^{\star}$ & $814.37 \pm 168.42^{\star}$ \\
Remodeling group & 51 & $51.26 \pm 15.47^{\star \#}$ & $142.59 \pm 41.27^{\star \#}$ & $1146.32 \pm 274.51^{\star \#}$ \\
F & & 102.817 & 240.522 & 319.018 \\
P & & 0.000 & 0.000 & 0.000 \\
\hline
\end{tabular}

To compared with the control group, ${ }^{*}, \mathrm{P}<0.05$; to compared with the non-remodeling group, ${ }^{*}, \mathrm{P}<0.05$.

Table 3 The correlation between serum indexes and ventricular remodeling in patients with heart failure after acute myocardial infarction

\begin{tabular}{lccccc}
\hline \multirow{2}{*}{ Index } & \multicolumn{2}{c}{ LVWT } & & \multicolumn{2}{c}{ LVMI } \\
\cline { 2 - 3 } \cline { 5 - 6 } & $r$ & $P$ & & $r$ & $P$ \\
\hline TIMP-3 & -0.485 & 0.001 & & -0.413 & 0.009 \\
CA125 & 0.512 & 0.000 & & 0.476 & 0.000 \\
NT-proBNP & 0.592 & 0.000 & 0.525 & 0.000 \\
\hline
\end{tabular}

The correlation between serum indexes and ventricular remodeling

In patients with heart failure following AMI, the serum TIMP-3 level was significantly negatively correlated with LVWT and LVMI ( $\mathrm{r}=-0.485,-0.413 ; \mathrm{P}=0.001,0.009)$. Furthermore, the serum CA125 level was significantly positively correlated with the LVWT and LVMI ( $\mathrm{r}=0.512$, 0.476; $\mathrm{P}=0.000,0.000)$, while the serum NT-proBNP level was significantly positively correlated with the LVWT and LVMI (r=0.592, 0.525; $\mathrm{P}=0.000,0.000 ;$ Table 3).

\section{Efficacy of serum indexes in predicting ventricular remodeling}

The area under the curve (AUC) for the serum TIMP-3 level alone in predicting ventricular remodeling was 0.740 , and the prediction sensitivity and specificity were $64.71 \%$ and $76.19 \%$, respectively. The AUC of the serum CA125 level alone in predicting ventricular remodeling was 0.759 , and the prediction sensitivity and specificity were $56.86 \%$ and $83.33 \%$, respectively. The AUC of the serum NTproBNP level alone in predicting ventricular remodeling was 0.697 , and the prediction sensitivity and specificity were $55.86 \%$ and $80.95 \%$, respectively. The AUC of the combination of serum TIMP-3, CA125, and NT-proBNP

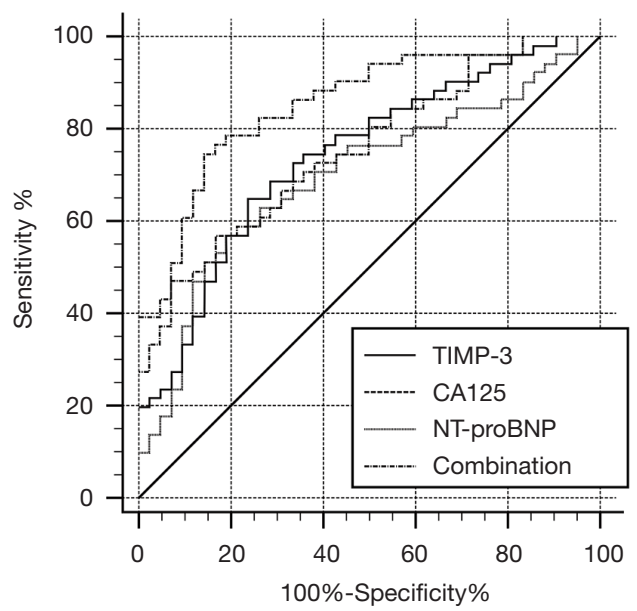

Figure 1 The ROC curve of serum indices in predicting ventricular remodeling. ROC, receiver operator characteristic.

levels in predicting ventricular remodeling was 0.850 , and the prediction sensitivity and specificity were $74.51 \%$ and $87.71 \%$, respectively. Therefore, combined prediction demonstrated the best sensitivity and specificity (Figure 1; Table 4).

\section{Logistic regression analysis of the ventricular remodeling}

Logistic regression analysis revealed that the lower the serum TIMP-3 level and the higher the serum CA125 and NT-proBNP levels, the greater the likelihood of ventricular remodeling. Therefore, TIMP-3, CA125, NT-proBNP, and Killip grade were risk factors for ventricular remodeling (Table 5).

\section{Discussion}

Heart failure represents the end stage of AMI, and 
Table 4 The comparison of efficiencies of serum indexes in predicting ventricular remodeling

\begin{tabular}{llcccc}
\hline Index & AUC & $95 \% \mathrm{Cl}$ & $\mathrm{P}$ & Sensitivity (\%) & Specificity (\%) \\
\hline TIMP-3 & 0.740 & $0.639-0.826$ & $<0.0001$ & 64.71 & 76.19 \\
CA125 & 0.759 & $0.659-0.841$ & $<0.0001$ & 56.86 & 83.33 \\
NT-proBNP & 0.697 & $0.593-0.788$ & 0.0004 & 55.86 & 80.95 \\
Combined prediction & 0.850 & $0.761-0.915$ & $<0.0001$ & 74.51 & 87.71 \\
\hline
\end{tabular}

Table 5 The logistic regression analysis of influence factors for ventricular remodeling

\begin{tabular}{lccc}
\hline Index & OR & $95 \% \mathrm{Cl}$ & $\mathrm{P}$ \\
\hline TIMP-3 & 1.451 & $1.123-1.875$ & 0.011 \\
CA125 & 1.267 & $1.098-1.462$ & 0.023 \\
NT-proBNP & 1.184 & $1.032-1.358$ & 0.036 \\
Killip grade & 2.014 & $1.732-2.342$ & 0.000 \\
\hline
\end{tabular}

ventricular remodeling is the main pathological basis of heart failure following AMI. Ventricular remodeling includes extra-cardiomyocyte collagen deposition, apoptosis, volume overload, and cardiomyocyte hypertrophy and occurs throughout the entire process of the occurrence and development of heart failure. Timely prediction and interruption of ventricular remodeling are important in the prevention and treatment of heart failure (8). MMPs are a large family of enzymes that degrade various extracellular proteins. MMPs are involved in several pathophysiologies, including angiogenesis, malignant tumor infiltration and metastasis, and atherosclerosis; in addition, they play a role in tissue remodeling by degrading fibrous collagen (9). It has been found that the polymorphism of the MMP gene promoter region functional sequence, particularly MMP-3, plays an active role in the pathogenesis of coronary heart disease and is involved in myocardial infarction and coronary atherosclerotic plaque (10). In one animal experiment, MMP-3 expression was shown to be upregulated in the myocardial interstitium of mice with heart failure, wherein it promoted myocardial remodeling and accelerated heart failure (11). TIMP is a type of MMP inhibitor, and MMP/TIMP imbalance can cause abnormal extracardiomyocyte collagen accumulation, which leads to ventricular stiffening and myocardial fibrosis and prevents myocardial relaxation and contraction. TIMPs can block MMPs from binding to their substrates and inhibit their activity, which subsequently delays the progression of ventricular remodeling $(12,13)$. TIMP-3 is a member of the TIMP family and is a specific non-soluble protein that inhibits MMP activity. It has been shown that the activity of gelatinase increases and TIMP-3 level decreases at the end stage of human heart failure, which suggests that TIMP-3 may inhibit myocardial remodeling (14). The results of the present study found that the serum TIMP-3 level was significantly lower in the ventricular remodeling group than in both the non-ventricular remodeling and control groups; furthermore, it was shown to be significantly negatively correlated with the LVWT and LVMI. Such findings indicate that serum TIMP-3 is closely correlated with ventricular remodeling in patients with heart failure following AMI. CA125 mainly comes from epithelial cells. A number of clinical trials have found abnormal increases in the serum CA125 concentration to be associated with heart failure (15). The results of the present study revealed that serum CA125 levels were significantly higher in the ventricular remodeling group than in both the non-ventricular remodeling and control groups; in addition, serum CA125 levels were found to be significantly positively correlated with the LVWT and LVMI. Such findings indicate that increases in the serum CA125 level correlate with ventricular remodeling. The reasons for the abnormal increase in CA125 level are as follows: the overexpression of proto-oncogenes in serum of patients with heart failure increases the secretion of CA125 and promotes myocardial remodeling. Furthermore, numerous inflammatory factors are secreted during heart 
failure, which in turn increase the secretion of CA125, increasing the left ventricular filling pressure and changing the structure. Luo et al. found that the serum CA125 level in diastolic heart failure increased as the cardiac grade increased and was correlated with heart function indicators. Such findings indicate that the serum CA125 level correlates closely with the severity of heart failure and ventricular remodeling and can serve as a diagnostic and evaluation index for heart failure (16).

NT-proBNP is mainly synthesized by cardiomyocytes, and an elevated expression of NT-proBNP can be used as a monitoring index for cardiac dysfunction and prognosis. As early as the $21^{\text {st }}$ century, the European Association of Cardiology included NT-proBNP in the guidelines for the diagnosis and treatment of heart failure, which are widely used in ventricular function evaluation (17). However, the predictive value of NT-proBNP in ventricular remodeling remains controversial. Yang et al. revealed that serum NTproBNP levels were significantly higher in AMI patients with ventricular remodeling than in AMI patients without ventricular remodeling. This suggests that the serum NTproBNP level is a sensitive index for predicting ventricular remodeling in patients with AMI (18). The present study found serum NT-proBNP levels to be significantly higher in the ventricular remodeling group than in the non-ventricular remodeling group; furthermore, NTproBNP levels were significantly positively correlated with the LVWT and LVMI. This suggests that serum NT-proBNP correlates with ventricular remodeling in patients with heart failure following AMI, which is in line with conclusions reported by Yang et al. Furthermore, Wu et al. found that serum NT-proBNP was not correlated with left ventricular remodeling following percutaneous coronary intervention (PCI) in patients with acute ST segment elevation myocardial infarction and had no independent predictive value. Increases in its expression may correlate with decreases in the left ventricular ejection fraction, which could be the result of different diagnostic methods (19). In the present study, the value of serum TIMP-3, CA125, and NT-proBNP in predicting ventricular remodeling was analyzed. The results showed that serum TIMP-3, CA125, and NT-proBNP have independent predictive value in predicting ventricular remodeling in patients with heart failure following AMI. Though their sensitivity in independent prediction remains poor, the predictive sensitivity of the combination of TIMP-3, CA125, and NT-proBNP was found to be significantly increased. It has been shown that the Killip grade correlates with poor prognosis in patients with AMI. In the present study, $50.98 \%$ of 51 patients with ventricular remodeling were patients at Killip IV. The logistic regression analysis revealed that TIMP-3, CA125, NT-proBNP, and the Killip grade were all risk factors for ventricular remodeling.

The present study has several limitations. First, the study was a single-center clinical study with a small sample size; therefore, there is a need to increase the sample size and conduct additional multicenter clinical studies. Second, the prognostic role of TIMP-3, CA125, and NT-proBNP in patients with heart failure after AMI was not identified in this study. Further studies are needed to investigate the prognostic role of these indicators.

\section{Conclusions}

In summary, serum levels of TIMP-3, CA125, and NTproBNP can independently predict ventricular remodeling in patients with heart failure following AMI. These three indicators were found to have high specificity and low sensitivity. However, the AUC of the combination of these three indicators was higher than that of the three indicators alone in terms of predicting ventricular remodeling, and in combination, the sensitivity and specificity of prediction was also increased. This suggests that the early combined detection of serum TIMP-3, CA125, and NT-proBNP levels can improve the accuracy of predicting ventricular remodeling and are helpful in the early prevention and treatment of heart failure following AMI.

\section{Acknowledgments}

Funding: The study was supported by Hebei Province Medical Science Research Key Project Plan (No. 20180163).

\section{Footnote}

Reporting Checklist: The authors present the study in accordance with the TRIPOD reporting checklist. Available at http://dx.doi.org/10.21037/cdt-20-399

Data Sharing Statement: Available at http://dx.doi. org/10.21037/cdt-20-399

Conflicts of Interest: All authors have completed the ICMJE uniform disclosure form (available at http://dx.doi. 
org/10.21037/cdt-20-399). The authors have no conflicts of interest to declare.

Ethical Statement: The authors are accountable for all aspects of the work in ensuring that questions related to the accuracy or integrity of any part of the work are appropriately investigated and resolved. The study was conducted in accordance with the Declaration of Helsinki (as was revised in 2013). The study was approved by institutional ethics board of Hebei General Hospital [2017 Scientific Research Proceedings No. (144)], and informed consent was provided by all patients.

Open Access Statement: This is an Open Access article distributed in accordance with the Creative Commons Attribution-NonCommercial-NoDerivs 4.0 International License (CC BY-NC-ND 4.0), which permits the noncommercial replication and distribution of the article with the strict proviso that no changes or edits are made and the original work is properly cited (including links to both the formal publication through the relevant DOI and the license). See: https://creativecommons.org/licenses/by-nc-nd/4.0/.

\section{References}

1. Venkatesan C, Mishra A, Morgan A, et al. Outcomes trends for acute myocardial infarction, congestive heart failure, and pneumonia, 2005-2009. Am J Manag Care 2016;22:e9-17.

2. Hanejin M, Ma YH, Wang HY, et al. Advances in the pathophysiology and pathogenesis of chronic heart failure. Chin J Integr Med Cardio 2016;14:1349-52.

3. Li GW, Li S. The effect of matrix metalloproteinase -3 gene transfer on myocardial infarction in rats. Chin J Mod Med 2012;22:1-6.

4. Zhuang J, Faggiano P, Li Q, et al. Insights into the clinical implications of carbohydrate antigen 125 as a biomarker of heart failure: a meta-analysis and systematic review of published studies. J Cardiovasc Med (Hagerstown) 2014;15:864-72.

5. Ordu S, Ozhan H, Alemdar R, et al. Carbohydrate antigen-125 and $\mathrm{N}$-terminal pro-brain natriuretic peptide levels: compared in heart-failure prognostication. Tex Heart Inst J 2012;39:30-5.

6. Jin M. Guidelines for the diagnosis and treatment of acute ST elevation myocardial infarction. Chin J Cardiol 2015;38:675-90.

7. Ibanez B, James S, Agewall S, et al. 2017 ESC Guidelines for the management of acute myocardial infarction in patients presenting with ST-segment elevation. Eur Heart J 2018;39:119-77.

8. Konstam MA, Kramer DG, Patel AR, et al. Left ventricular remodeling in heart failure: current concepts in clinical significance and assessment. JACC Cardiovasc Imaging 2011;4:98-108.

9. Geng XL, Fan YC, Li GB, et al. Research progress of matrix metalloproteinase on ventricular remodeling after myocardial infarction and traditional Chinese medicine. Tianjin J Tradit Chin Med 2010;27:175-6.

10. Li R, Meng ZH. Progress of matrix metalloproteinase in coronary heart disease. Clinical Medicine 2017;37:123-7.

11. Zhang B, Ma JG, Wu G, et al. Effects of probucol on the expression of mmp-3, timp-3 and OPN in heart failure rats. Journal of Harbin Medical University 2015:115-20.

12. Lu J, Hao J, Du H, et al. Amlodipine and Atorvastatin Improved Hypertensive Cardiac Remodeling through Regulation of MMPs/TIMPs in SHR Rats. Cell Physiol Biochem 2016;39:47-60.

13. Braiek AB, Bruno B, Claudine D, et al. Gene polymorphism and circulating levels of matrix metalloproteinases (MMPs) and their tissue inhibitors (TIMPs) in coronary artery disease (CAD). Atherosclerosis 2017;263:e282.

14. Eckhouse SR, Purcell BP, McGarvey JR, et al. Local hydrogel release of recombinant TIMP-3 attenuates adverse left ventricular remodeling after experimental myocardial infarction. Sci Transl Med 2014;6:223ra21.

15. Ren GG. Research progress of correlation between CA125 and heart failure and congenital heart disease. Journal of Clinical Pediatric Surgery 2017;16:198-201.

16. Luo YS, Pan YZ. Correlation analysis of serum carbohydrate antigen 125 with brain natriuretic peptide precursor, left ventricular structure and cardiac function in patients with diastolic heart failure. South China Journal of Cardiovascular Diseases 2015;21:459-61.

17. Park CS, Park JJ, Oh IY, et al. Relation of Renal Function with Left Ventricular Systolic Function and NT-proBNP Level and Its Prognostic Implication in Heart Failure with Preserved versus Reduced Ejection Fraction: an analysis from the Korean Heart Failure (KorHF) Registry. Korean Circ J 2017;47:727-41.

18. Yang K, Chen G, Yang FY, et al. The predictive value of serum nt-probnp test for ventricular remodeling in patients with acute myocardial infarction. Chin J Mod 
Med 2014;24:54-7.

19. Wu XL, Jia YB, L JY, et al. The predictive value of cTnI, hs-crp and nt-probnp levels in left ventricular remodeling after elective PCI for acute st-segment elevation myocardial infarction. Chin J Integr Med Cardio 2013;11:672-3.

Cite this article as: Xie YT, Dang Y, Zhang FF, Zhang QH, Wu HB, Liu G. Combination of serum TIMP-3, CA125, and NT-proBNP in predicting ventricular remodeling in patients with heart failure following acute myocardial infarction. Cardiovasc Diagn Ther 2020;10(5):1184-1191. doi: 10.21037/ cdt-20-399 